\section{JACKKNIFE: A general-purpose package for generating multivariate jackknife analyses}

\author{
JOSEPH L. BALLOUN and A. BEN OUMLIL \\ University of Dayton, Dayton, Ohio
}

Two FORTRAN IV programs are described which facilitate multivariate jackknife analyses.

\section{Theory of Jackknife Methods}

A common problem in statistical analysis is that we know how to calculate a parameter estimate, $\hat{\theta}$, from sample data, but we do not know how to assess the variability of the estimate or how to make significance or confidence interval calculations (Tukey, 1980). When variability or significance or confidence interval estimates are available it is often assumed that the sample data are well conditioned (cf. Nie, Hull, Jenkins, Steinbrenner, \& Bent, 1975). For example, inferential tests in multiple regression analysis assume that the data contain no outliers, are drawn from a single population, are homoscedastic, are multivariately normal, and are free from collinearity, and that the associated correlation matrix is nonsingular for for inversion purposes. Often in multiple regression and other multivariate analysis methods there is doubt that these assumptions are sufficiently well met to allow adequate modeling of the data or statistical inferences by the usual procedures.

Many variables are far from normally distributed. For example, response latency, dichotomous variables, error scores, deviations from a social norm, and accident rates are usually not normally distributed. Normalizing transformations do not remedy all such problems. Correlation matrices are often not "well conditioned." For example, missing data in survey research often create ill-conditioned correlation matrices. Variables such as income, amount of rainfall, the weight of hogs, and response frequency often exhibit heteroscedasticity when correlated with other variables.

When there is no efficient way to calculate sampling variances, or when the available methods make implausible assumptions, there are general methods for calculating sampling variance, confidence intervals, or significance levels (e.g., double cross-validation). The jackknife method is the best such general method (Tukey, 1980). It removes bias proportional to the reciprocal of sample size $(N)$, tends to reduce estimate bias, and provides a means of estimating error variance and sig-

We are indebted to three anonymous reviewers who made valuable comments and suggestions on an earlier draft of this paper. J. L. Balloun is with the Department of Management and A. Ben Oumlil is with the Department of Marketing, both at the University of Dayton, Dayton, $\mathrm{OH} 45469$. nificance or confidence intervals when alternatives are unavailable or their assumptions are violated. The method is generally robust, but caution or data transformation may be necessary in special circumstances. Miller (1974), Mosteller and Tukey (1968), and Bissell (1977) provide further information on the rationale for the jackknife technique.

\section{Computational Method}

The computational method involves the following steps (Bissell, 1977; Tukey, 1980; symbols are defined in Table 1): (1) Calculate the parameter estimate, $\hat{\theta}$, based on all sample data. (2) Divide the data into $r$ stochastically equivalent subgroups. The $r$ subgroups will be of size $s$. In the program JACKKNIFE, $s=1, r=N$, and each observation is a separate subgroup. (3) Each subgroup is omitted from the data in turn. This results in $r$ partial or decremented estimates, $\hat{\theta}_{-j}$, each based on the remaining $r-1$ subgroups. (4) From each decremented estimate calculate a pseudovalue:

$$
\hat{\theta}_{*_{j}}=r \hat{\theta}-(r-1) \hat{\theta}_{-j} .
$$

(5) The jackknife estimate is the mean of the pseudovalues:

$$
\hat{\theta}_{*}=\frac{1}{r} \sum_{j=1}^{r} \hat{\theta}_{*_{j}}
$$

(6) The variance is calculated from the pseudovalues as if they were independent observations:

$$
\hat{s}_{\theta_{*}}^{2}=\frac{\sum_{j=1}^{r}\left(\theta_{*_{j}}-\theta_{*}\right)^{2}}{r-1} ;
$$

\begin{tabular}{|c|c|}
\hline Symbol & Meaning \\
\hline$\hat{\theta}$ & $\begin{array}{l}\text { A sample estimate of a population parameter calcu- } \\
\text { lated from the total sample }\end{array}$ \\
\hline$\hat{\theta}_{-j}$ & $\begin{array}{l}\text { The sample estimate when the jth subgroup is omitted } \\
\text { and the estimate is found from the remaining data }\end{array}$ \\
\hline$\hat{\theta}_{* j}$ & $\begin{array}{l}\text { A jackknifed "pseudovalue" for each subgroup, com- } \\
\text { puted as in Equation } 1\end{array}$ \\
\hline$\theta_{*}$ & The mean of the $\hat{\theta}_{k_{j}}$ values \\
\hline$s_{\theta_{*}}$ & The unbiased sample standard deviation of $\hat{\theta}_{* j}$ values \\
\hline$N$ & The sample size \\
\hline$\hat{\phi}$ & $\begin{array}{l}\text { A } \theta \text { estimate transformed to reduce possible nonnor- } \\
\text { mality or to avoid "out of range"' estimates. The sym- } \\
\text { bol may appear with various subscripts, as for } \theta\end{array}$ \\
\hline$r$ & Number of subgroups used \\
\hline$s$ & Size of each subgroup \\
\hline$k$ & Number of parameters to be estimated \\
\hline
\end{tabular}

Table 1

Jackknife Methods 
The standard error of estimate for $\theta$ is then

$$
S E_{\hat{\theta}_{*}}=\frac{S_{\theta_{*}}}{r} .
$$

(7) Where many parameters are estimated, the procedure will result in a new data matrix with $r$ rows and $k$ columns, where $r$ is the number of subgroups and $k$ is the number of estimated parameters. Various multivariate analyses can be applied to this new data matrix of jackknifed or decremented estimates.

\section{Discussion of Method}

In some instances the jackknife should not be used. It is pointless to develop jackknife estimates of the mean because the pseudovalues are the original data values. More complicated versions of the jackknife are needed for order or extreme value statistics (Bissell, 1977).

Normalizing transformations should be used on $\hat{\theta}, \hat{\theta}_{-j}$, $\hat{\theta}_{*_{j}}$, or $\hat{\theta}_{*}$ in appropriate circumstances. The transformations generally prevent a parameter estimate from falling outside the allowable range.

Thus, within program JACKKNIFE, standard deviations are transformed logarithmically:

$$
\hat{\phi}=\ln (\hat{\theta}+1),
$$

where $\hat{\theta}$ is the "unbiased" standard deviation estimate. Correlations are transformed by the Fisher $z$ ' transformation:

$$
\hat{\phi}=.5 \ln \frac{1+\hat{\theta}}{1-\hat{\theta}},
$$

where $\hat{\theta}$ is the Pearson sample correlation. Absolute errors in a multiple regression application of prediction may be transformed logarithmically:

$$
\hat{\phi}=\ln \left(\left|Y_{i}-\hat{Y}_{i}\right|+1\right),
$$

where $Y_{i}$ is the observed criterion score for the ith case, and $\hat{Y}_{i}$ is the corresponding predicted criterion score on the basis of the model developed from all other cases.

\section{Basic Logical Flow of Programs}

The raw data are input to program JACKKNIFE. Program JACKKNIFE prints the number of cases, means, standard deviations, and Pearson correlations for the input raw data. It produces three output disk files: a copy of the raw data; a "decremented" file containing the number of cases, standard deviations, and correlations with all cases and when each case is deleted; and a file containing jackknifed standard deviations and jackknifed correlations.

Program JACKREG reads the raw data file and the decremented file written by program JACKKNIFE. It prints the ordinary least squares solution for each model specified. It writes a disk file which contains the jackknifed intercept and slopes for each model, plus the logarithm of the unbiased error of prediction for each case.

A standard package, for example, the Statistical Package for the Social Sciences (Nie, Hull, Jenkins, Steinbren- ner, \& Bent, 1975), is used to further process the outputs from JACKREG.

A similar logical flow can be used for other types of possible multivariate jackknife analyses.

\section{Program JACKKNIFE Description}

The purpose of the JACKKNIFE program is to provide general-purpose jackknife statistics useful for many different statistical applications. The JACKKNIFE program calculates the following statistics from an input data set: (1) the number of valid cases, mean, and standard deviation for each variable; (2) the Pearson correlations among all variables; (3) the number of cases, mean, and standard deviation for each variable when each case, one at a time, is deleted from the sample; (4) the Pearson correlations among all variables when each case, one at a time, is deleted from the sample; (5) the jackknifed natural logarithms of one plus the standard deviations for each variable for each case; (6) the jackknifed Fisher $z^{\prime}$ transformations of the correlation coefficients for each case.

These statistics are useful because many frequently used multivariate statistical techniques depend upon estimates of population means, standard deviations, and correlations. The calculated statistics are written to disk files for later access by other computer programs.

Input. The user prepares a set of control cards that describe the number of cases, the number of variables, the logical input unit for the raw data, variable names if desired, and the input format of the raw data. These control cards are followed by the raw data in card image form (unless the raw data are in another disk file). Missing data are followed and are identified on output.

Output. Three files are written by JACKKNIFE. The first is a copy of the raw input data file. The second contains the jackknifed standard deviations and correlation coefficients for each case. Missing data are identified on output. The first logical record in the third file contains the total sample number of cases, means, standard deviations for each variable, and the intercorrelations among all variables. The next $N$ records in this file have the same logical structure as the first record and contain the means, standard deviations, and intercorrelations that result when each case is deleted from the sample one at a time.

Limitations. A provisional means algorithm is used to increase computational accuracy. Data from the first input logical record are used for temporary means. The number of variables is currently restricted to a maximum of 50 . Capacity can be increased by straightforward changes to the source code; thus, more than 50 variables can be accommodated if the user has access to greater time or memory. In some applications the user can reduce the number of variables in the problem by preliminary factor analysis or related procedures.

\section{Program JACKREG Description}

A program has been written to generate jackknife multiple regression analyses. JACKREG uses the same ra- 
tionale as program JACKKNIFE, and its main input consists of the outputs from program JACKKNIFE.

Input. The user prepares a set of control cards that describe a problem or task title, the number of cases, and the number of variables. JACKREG does not perform stepwise multiple regression. Instead, the user specifies one or more multiple regression models to be analyzed. JACKREG reads in two disk files prepared by program JACKKNIFE. One input file contains the number of cases, means, and standard deviations for each variable; the other is a copy of the raw data. The organization of these files is explained in detail in the user's guide for the JACKKNIFE program. Missing data are allowed and are identified on output.

Output. JACKREG prints the ordinary least squares regression results. The jackknifed intercept and slopes and the natural logarithm of the decremented standard error of prediction (plus one) for each case are written in card image format to a disk file. The jackknifed slopes are output.

The user may then use standard statistical utility programs to access this output disk file for further analyses. Such subsequent analyses might include the following topics: (1) robust and unbiased estimates of intercept or slopes, (2) unbiased estimates of multiple correlation, (3) significance tests for parameter estimates when classical assumptions are violated, (4) description of param- eter estimate distributions and distribution normality tests, (5) correlations among parameter estimates, (6) detection of outliers in the data.

\section{Limitations}

JACKREG has the same limitations as program JACKKNIFE.

\section{Availability}

Program listings and users' manuals are available free of charge from the authors.

\section{REFERENCES}

Bissell, A. F. (1977). The jackknife. Bulletin in Applied Statistics, 4, 55-64.

Miller, R. G. (1974). The jackknife: A review. Biometrika, 61, 1-17.

Mosteller, F., \& TukEY, J. W. (1968). Data analysis including statistics. In G. Lindzey \& E. Aronson (Eds.), The handbook of social psychology (Vol. 2). Reading, MA: Addison-Wesley.

Nie, N. H., Hull, C. H., Jenkins, J. G., STeinbrenner, K., \& Bent, D. H. (1975). Statistical package for the social sciences (2nd ed., pp. 340-342). New York: McGraw-Hill.

TUKEY, J. W, , (1980). Methodological comments focused on opportunities. In P.R. Monge \& J. N. Capella (Eds.), Multivariate techniques in human communication research (pp. 490-528). New York: Academic Press.

(Revision accepted for publication January 21, 1986.) 